\title{
Esclerosis múltiple de alta actividad: ¿se puede iniciar precozmente el tratamiento con drogas de alta eficacia?
}

\section{Highly active multiple sclerosis: is it possible to start early therapy with highly efficacious drugs?}

Correspondencia

Charles Huamaní Saldaña

huamani_ca@hotmail.com

Recibido: 04/06/2017

Arbitrado por pares

Aprobado: 06/09/2017

Citar como: Huamani C, Rojas E, Inca J. Esclerosis múltiple de alta actividad: ¿se puede iniciar precozmente el tratamiento con drogas de alta eficacia? Acta Med Peru. 2017;34(4):301-8

\author{
Charles Huamaní1 ${ }^{1 \mathrm{bb}}$, Edgard Rojas ${ }^{1 c}$, José Inca ${ }^{1 c}$ \\ 1 Servicio de Neurología, Hospital Nacional Guillermo Almenara. Lima, Perú. \\ a Médico epidemiólogo, b Residente de neurología, c Médico neurólogo
}

\section{RESUMEN}

La esclerosis múltiple (EM) es una enfermedad crónica del sistema nervioso central (SNC) que desarrolla cuadros clínicos muy variados, puede progresar con brotes, dejar secuelas entre ellos, y tener un lento curso discapacitante. Sin embargo, en algunas personas los brotes tienen compromiso múltiple del SNC, rápido deterioro de la discapacidad, y otros factores de riesgo de mal pronóstico de la enfermedad. Aunque no hay consenso sobre su definición, estas características indican que estamos ante una EM de alta actividad. Poder identificar la EM de alta actividad de forma precoz nos permite individualizar su tratamiento y usar drogas de alta eficacia. Nosotros describimos la dificultad para conciliar una definición apropiada de EM de alta actividad y la necesidad de usar de forma precoz drogas de alta eficacia en aquellas EM de alta actividad que se presentan desde el inicio de la enfermedad.

Palabras clave: Esclerosis múltiple; Personas con discapacidad; Enfermedad crónica (fuente: DeCS BIREME).

\section{ABSTRACT}

Multiple sclerosis (MS) is a chronic central nervous system (CNS) disease that may have variable clinical manifestations, it may progress as flares, leaving sequels between such flares, and have a slow disabling course. However, in some persons, MS flares lead to marked CNS involvement, rapid disability progression, and to other factors that lead to a poor prognosis for this disease. Although there is no consensus for a definition, these features may indicate that the condition is a highly active form of MS. Being able to early identify highly active MS would allow us to have individualized therapy and use highly efficacious drugs. In this paper we describe how difficult it is to have an adequate definition of highly active MD, and the need for early start using highly efficacious drugs for these highly active forms of MS that occur as early flares of the disease.

Keywords: Multiple sclerosis; Disabled persons; Chronic disease (source: MeSH NLM). 


\section{INTRODUCCIÓN}

La esclerosis múltiple (EM) es una enfermedad crónica autoinmune que afecta cualquier región del sistema nervioso central (SNC), por lo que el compromiso clínico es variado, pudiendo presentarse con déficit motor, sensitivo, sensorial, autonómico, cognitivo, entre otros. Afecta con frecuencia a población joven en plena actividad productiva (20-40 años), generando principalmente discapacidad. En varios países de Europa y Norteamérica, la prevalencia es mayor a 100 casos por 100000 habitantes, pero para países sudamericanos se encuentra entre 10 a 20 casos por 100000 habitantes ${ }^{[1,2]}$. Sin embargo, con el mayor acceso a la resonancia magnética (RM), cada vez son más los casos diagnosticados, cuyas características clínicas en Perú son similares a las descritas en la literatura mundial ${ }^{[3]}$.

Actualmente la EM puede ser diagnosticada de forma precoz, incluso puede sospecharse en eventos clínicos aislados. Estos escenarios han permitido diseñar estudios para demostrar que el uso precoz de "terapias modificadoras de la enfermedad" tienen un mejor resultado en el control de los brotes y progresión de la discapacidad; siendo esta ahora una recomendación común ${ }^{[4]}$. Las terapias en EM se dividen en drogas de primera línea, que incluye los interferones o el acetato de glatiramer, entre otros; y para pacientes con presentaciones agresivas o que presentan fracaso al tratamiento, se puede emplear terapias de segunda línea o alta eficacia (Tabla 1) ${ }^{[5,6]}$.

En los establecimientos públicos del Perú y en otros países vecinos no se dispone de drogas de alta eficacia o su acceso requiere primero, el uso de terapias de primera línea por 6 a 12 meses y demostrar que francamente se ha fracasado con las drogas de primera línea. Ello debido a que el uso de drogas de alta eficacia se suele indicar solo cuando hay falla terapéutica y no cuando se está en formas clínicas con alta actividad o agresividad desde el inicio de la enfermedad. Nosotros presentamos una revisión de los conceptos de "alta actividad" en EM, discutiendo sobre la falta de consenso, pero resaltando la necesidad iniciar precozmente su tratamiento con drogas de alta eficacia.

\section{CONSIDERACIONES PARA EL DIAGNÓSTICO Y ESTABLECER LA DISCAPACIDAD DE LA ENFERMEDAD}

Las formas clínicas de la EM incluyen la forma remitenterecurrente (EM-RR) y la forma progresiva ${ }^{[7]}$. Durante el curso de la enfermedad aparecerán brotes, nuevas lesiones, generará discapacidad y se valorará la actividad de la enfermedad.

Los "brotes" son la expresión clínica de una lesión del SNC, que se define como la aparición de algún déficit del SNC que dura más de 1 día, pudiendo ser motor, sensitivo, sensorial, cerebeloso, cognitivo, esfinteriano, etc. Con frecuencia remiten dejando poca o ninguna secuela, a la expectativa de un nuevo brote que debe ocurrir con un intervalo mínimo de 1 mes. Debe ocurrir en ausencia de un proceso infeccioso o fiebre. Se recomienda describir el número de brotes por año.

Las “lesiones" en EM son imágenes visibles en RM, con frecuencia ovoideas, siempre hiperintensas en las secuencias T2/FLAIR e hipointensas en T1 (Figura 1). Las lesiones antiguas pueden desaparecer, pero si persisten hipointensas en T1 por más de 6 meses se conocen como "agujeros negros". Las lesiones nuevas o gadolinio positivas, suelen generar imágenes con realce en anillo incompleto y realzar contraste hasta por tres meses. Es

Tabla 1. Medicamentos aprobados para esclerosis múltiple y disponibles en el Perú.

\begin{tabular}{|c|c|c|c|}
\hline Nombre & Eficacia* & Administración & Principales efectos adversos \\
\hline \multicolumn{4}{|c|}{ Primera línea o moderada eficacia } \\
\hline Interferon b1a sc & $32 \%$ & SC 3 veces/sem & $\begin{array}{l}\text { Lesiones dérmicas incremento de } \\
\text { enzimas hepáticas }\end{array}$ \\
\hline Interferon b1a im & $32 \%$ & IM semanalmente & Incremento de enzimas hepáticas \\
\hline Acetato glatiramer $20 \mathrm{mg}$ & $29 \%$ & SC diario & Lipodistrofias, alergias \\
\hline \multicolumn{4}{|l|}{ Segunda línea o alta eficacia } \\
\hline Fingolimod & $55-60 \%$ & VO diario & $\begin{array}{l}\text { Linfopenia, infecciones, bloqueo } \\
\text { auriculo-ventricular, edema de mácula }\end{array}$ \\
\hline Natalizumab & $68 \%$ & EV mensual & $\begin{array}{l}\text { Leucoencefalopatía multifocal } \\
\text { progresiva }\end{array}$ \\
\hline Alentuzumab & $70 \%$ & $\begin{array}{l}5 \text { días de infusión, repetidas } \\
\text { al año siguiente por } 3 \text { días }\end{array}$ & Tiroideopatías, infecciones, púrpura \\
\hline
\end{tabular}

* Eficacia: Porcentaje de reducción del riesgo relativo de brotes. 


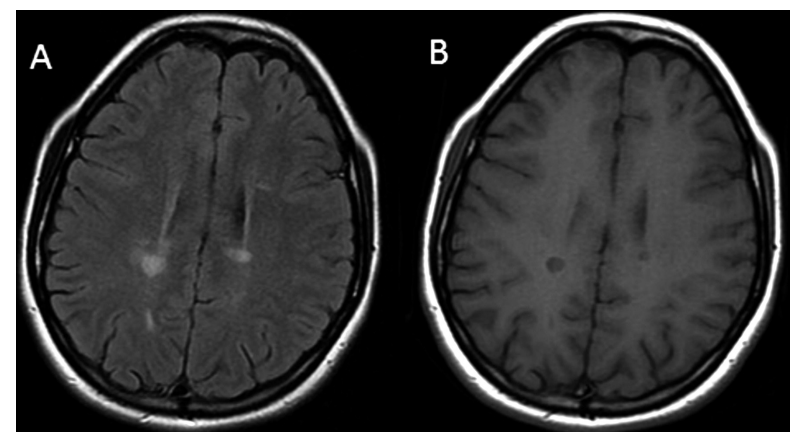

Figura 1. Imágenes de resonancia magnética en esclerosis múltiple.

frecuente la presencia de nuevas lesiones sin brotes (actividad asintomática), al contrario, es raro un real brote sin la evidencia de una nueva lesión en RM.

La "discapacidad" valora el compromiso motor, sensitivo, visceral, cognitivo, entre otras áreas producido por los brotes. Se emplea la EDSS (Expanded Disability Status Scale), que va desde 0 para personas sin discapacidad, hasta 10 para la muerte por EM ${ }^{[8]}$. Llegar a un EDSS mayor de 6 (requerir ayuda constante para caminar más de 100 metros) indica discapacidad severa ${ }^{[9]}$.

La "progresión" se refiere al incremento de la discapacidad independiente de la presencia de brotes, que debe ser valorada anualmente a través de parámetros objetivos como la EDSS. Cuando desde un inicio se presenta discapacidad que va en progreso, sin poder identificar con claridad los brotes, estamos ante la forma primaria progresiva (EM-PP). En cambio, cuando una forma EM-RR progresa sin la necesidad de nuevos brotes, cambia a una forma secundaria progresiva (EM-SP), esta transición es usualmente gradual. Operativamente, la progresión se define como un incremento anual de 1,5 puntos en EDSS si el puntaje de base era 0, de 1 punto si la base era 1 a 5 puntos, o un incremento de 0,5 puntos si se tenía de base más de 5 puntos.

La "actividad" se refiere a la presencia no controlada de la enfermedad, evaluada a través de nuevos brotes, nuevas lesiones en RM o incremento de la discapacidad en un periodo de seguimiento. La ausencia de novedades en las tres (broteslesiones-discapacidad) define la "no evidencia de actividad" (NEDA-3) de la enfermedad, actualmente también se considera la ausencia de atrofia cerebral (NEDA-4) ${ }^{[10]}$. Es aplicable tanto para la forma EM-RR como para las formas progresivas.

La EM-RR se caracteriza por su presentación en brotes, para el diagnóstico se precisa al menos dos brotes clínicos y la visualización de lesiones en RM cumpliendo los criterios de diseminación en espacio y tiempo. La diseminación en espacio requiere la presencia de lesiones en dos áreas distintas del SNC, que es dividida en cinco: nervio óptico, lesiones periventriculares (donde debe haber de tres a más lesiones), cortico-yuxtacorticales, infratentoriales, y medulares (Figura 1). Para diseminación en tiempo se requiere evidenciar en la primera
RM lesiones que captan contraste al mismo tiempo de otras que no lo hacen, o durante el seguimiento, ver la aparición de nuevas lesiones en la RM de control ${ }^{[11]}$.

Cuando se presenta solo un brote junto a lesiones en RM que no completan los criterios de diseminación en tiempo y espacio se denomina "síndrome clínico aislado". Mientras que si incidentalmente se identifican en RM lesiones compatibles con EM en ausencia de actividad clínica se denomina "síndrome radiológico aislado".

\section{TERAPIAS MODIFICADORAS DE LA ENFERMEDAD}

La EM es una enfermedad autoinmune que ha presentado un gran avance en las opciones terapéuticas en las últimas décadas, con medicamentos que disminuyen la actividad antiinflamatoria hasta anticuerpos neutralizantes, lo cual ha causado un impacto importante en la modificación del curso de la enfermedad.

El inicio del tratamiento en la EM incluye los síndromes clínicos aislados con alto riesgo de conversión a EM ${ }^{[12]}$, y la EM con un EDSS menor de 6,5. El objetivo terapéutico más ambicioso es conseguir la NEDA, que se consigue en el $12 \%$ de los pacientes tratados con Interferón a los 5 años ${ }^{[13]}$. Los estudios iniciales realizados con Interferón, mostraron que luego de 15 años de seguimiento, la presencia de al menos 2 lesiones que realzan gadolinio durante los primeros 2 años de tratamiento, son altamente predictivos de un mal curso de la enfermedad ${ }^{[14,15]}$. Por ello, son varias las guías que recomiendan la pronta optimización del tratamiento, pues se traduce en una reducción de la discapacidad y el número de brotes, en especial dentro de los dos primeros años de enfermedad o cuando la discapacidad aún es baja ${ }^{[4,14,16]}$.

Las terapias modificadoras de enfermedad en EM han sido agrupadas en dos: terapias de primera línea o de moderada eficacia; y terapias de segunda línea o de alta eficacia ${ }^{\left[{ }^{[6} \text { (Tabla }\right.}$ 1). Hay presentaciones de EM-RR que cursan con poca actividad, por lo que se pueden controlar adecuadamente con las primeras opciones terapéuticas, que tienen una eficacia menor al 50\% en el control anual de brotes, aunque sus efectos adversos son transitorios y poco severos que generalmente son bien tolerados. En contraparte, presentaciones de EM con mayor actividad o que fracasan a la terapéutica inicial requieren drogas de alta eficacia, que disminuyen hasta en $70 \%$ el número anual de brotes, pero los efectos adversos pueden ser severos, como por ejemplo, la encefalopatía multifocal progresiva secundaria al virus JC.

El tiempo que se requiere para que las drogas inicien el proceso de control de la enfermedad, medido a través de un menor número de brotes o progresión de discapacidad, es entre 3 a 6 meses ${ }^{[17]}$. Los interferones, por ejemplo, requieren de la administración continua durante 6 meses para ser efectivas, antes de ello, se podría considerar al paciente "desprotegido" y no podríamos atribuir que los brotes que presente sean por 
falla del tratamiento. En cambio, las drogas de segunda línea muestran mejores resultados de una forma más precoz, por ejemplo, Fingolimod mostró reducción del riesgo de progresión de discapacidad desde los 3 meses de tratamiento ${ }^{[18]}$. A su vez, Alemtuzumab mostró mejores resultados e inclusive mostró mejoría clínica al compararse con Interferón, siendo significativa la diferencia desde el tercer mes de uso ${ }^{[19]}$.

También, no toda falla terapéutica es secundaria a una alta actividad de la enfermedad, pero una alta actividad sí se puede acompañar de una mala respuesta terapéutica a las drogas de primera línea. Por tanto, son los clínicos quienes deben valorar no solo la respuesta terapéutica y anticiparse a una falla al tratamiento, sino también el equilibrio entre la eficacia terapéutica y sus efectos adversos, eligiendo la mejor opción según la necesidad del paciente dada la presentación de su enfermedad, con el fin de disminuir los riesgos y mejorar la calidad de vida ${ }^{[20]}$.

En el caso de las formas progresivas, hasta el 2016 no se disponía de ningún tratamiento siendo el último fármaco en ser introducido el Ocrelizumab ${ }^{[21] .}$

\section{DESCRIBIENDO A LA ESCLEROSIS MÚLTIPLE SEGÚN SU NIVEL DE ACTIVIDAD}

No existe una definición clara o consenso que divida la EM según su nivel de actividad o progresión de discapacidad ${ }^{[22]}$. Sin embargo, se sabe que la EM es una enfermedad pleomórfica, con presentaciones de menor o mayor actividad, por ello se acuñaron términos como EM benigna, muy activa, con alta actividad, agresiva, y maligna o fulminante. La subdivisión que realizaremos es para las formas de EM-RR.

El término EM-RR "benigna" ha sido discontinuado y debe ser evitado. Anteriormente esta era definida como EM-RR con un EDSS de 3 puntos a los 10 años del inicio de la enfermedad. Pero al completar los 20 años de seguimiento, solo el 52\% seguía siendo benigna, el $21 \%$ progresó al uso de bastón (EDSS < = 6), y el $23 \%$ se convirtió en EM-SP, sin poder identificar un claro factor que prediga la benignidad del curso en EM ${ }^{[23]}$. Por lo que no se puede considerar ninguna forma benigna y no se debe plantear retirar el tratamiento.

De otro lado, se sabe de formas de EM "fulminantes" o "malignas", términos reservados para las variantes de Marburg o la esclerosis concéntrica de Baló ${ }^{[24]}$, que son monofásicas y llevan a la muerte en muy corto plazo, estas formas no serán tocadas en este documento.

Sobre las formas de EM “agresivas", en el 2013 un estudio dirigido por la University of British Columbia propuso tres escenarios ${ }^{[25]}$ : 1) presentar un EDSS de 6 o más a los 5 años del inicio de la enfermedad; 2) presentar un EDSS de 6 o más antes de los 40 años de edad; y 3 ) cambiar a una forma EM-SP dentro de los 3 años de iniciada la enfermedad; en las dos primeras definiciones se podían incluir formas progresivas, en la tercera solo se incluían los que iniciaban como EM-RR. El problema de esta propuesta radica en que al Ilegar a 6 en el EDSS o pasar a una EM-SP, reduce las opciones terapéuticas e inclusive se podrían discontinuar.

Con la intención de hacer que la definición ayude cuando aún se pueda hacer cambios en el tratamiento, otros autores han planteado otras características para EM "agresivas", como son: 1) EDSS de 4 en los primeros 5 años de tratamiento, 2) Pobre respuesta terapéutica luego de 1 año de instalada (no debidas a intolerancia), 3) durante el año de tratamiento, presentar 2 o más brotes con resolución incompleta, o 2 o más lesiones en T2/FLAIR o captadoras de contraste ${ }^{[22,26]}$. Aunque en menor medida, esta propuesta presenta limitaciones parecidas a la anterior.

Además de varias definiciones de "agresividad", también está la definición de "alta actividad", que ha tenido un fin más práctico. En los ensayos clínicos donde se valoró la eficacia de las drogas de alta actividad se incluyeron definiciones para pacientes que ya habían recibido tratamiento y que habían presentado falla terapéutica o actividad de la enfermedad a pesar de la terapia; así como para pacientes que no habían recibido tratamiento, quienes debían cumplir con los siguientes requisitos: 1) Presentar dos a más brotes el año previo al estudio, y 2) Tener una o más lesiones que realcen gadolinio al momento de ingresar al estudio ${ }^{[27,28]}$. Sin embargo, esta definición operativa incluiría una proporción relativamente grande de pacientes, pues es común apreciar al inicio de la enfermedad un alto número de lesiones hiperintensas en T2 o FLAIR en la RM, inclusive con agujeros negros y lesiones captadoras de contraste; además de haberse diseñado con fines de ensayos clínicos y poder tener interpretaciones de fin comercial.

La definición comúnmente aceptada para EM de "alta actividad", aunque aún vaga, es la rápida o temprana discapacidad debido a frecuentes brotes con incompleta recuperación, y un elevado número de lesiones objetivables en la RM, independientemente si se encuentra o no en tratamiento con drogas de primera línea ${ }^{[6,22,26]}$. La Association of British Neurologists agrega la "presencia de al menos 9 lesiones en la RM cerebral" ${ }^{[6]}$. Permaneciendo la definición de "alta actividad" aún a discusión.

Son varios los estudios que han identificado factores de riesgo para un mal pronóstico de la EM y nos indican que estaríamos ante formas de "alta actividad", descritas en la Tabla 2. Por ejemplo, un estudio que siguió durante 7 años pacientes con EM de varias regiones, encontró que ninguno de los hispanos - latinos conseguía estar libre de actividad de la enfermedad (NEDA) inclusive a los 2 años ${ }^{[13]}$. Aunque cualquiera de los descritos en la Tabla 2 es un factor de riesgo, debemos valorarlos en conjunto para definir si estamos ante una EM de alta actividad, pues solo el número de brotes o solo el número de lesiones han mostrado datos controversiales sobre la actividad de la enfermedad ${ }^{[14]}$. Por todo, son dos los escenarios para la EM de "alta actividad": 
Tabla 2. Factores de riesgo asociados con mal pronóstico en esclerosis múltiple ${ }^{[22,26]}$.

\section{Factores de riesgo}

\section{Demográficos \\ Inicio a una edad mayor de 40 años \\ Varón \\ De procedencia africana, hispánico o latino}

\section{Características clínicas}

Severidad de los brotes

- Brote moderado o severo (>= 1 punto del EDSS o que requiera hospitalización)

Tipo de ataque

- Multifocal (de forma simultánea en más de un área anatómica)

- Con parcial o incompleta recuperación a pesar del uso de esteroides

- Que afecte funciones cognitivas, motoras, cerebelosas, medular o esfinterianas.

Frecuencia

- Brotes frecuentes en los primeros 2 a 5 años

- Intervalo interataque corto

\section{Curso de la enfermedad}

Rápida progresión de la discapacidad (EDSS de 3 dentro de los 5 primeros años)

Inicio con formas progresivas

\section{Características de la resonancia magnética}

Al inicio

- Múltiples lesiones en T2

- Más de 2 lesiones que realzan con gadolinio

- Presencia de lesiones hipointensas en T1 (agujeros negros)

- Atrofia cortico-subcortical discernible de forma temprana

- Mayor número de lesiones infratentoriales

- Lesiones medulares

Durante el seguimiento

- Presencia de nuevas lesiones en T2

- Más de una nueva lesión que realza con gadolinio

- Lesiones medulares

EDSS: Expanded Disability Status Scale

Escenario 1: Durante el seguimiento de la enfermedad. Inician como EM con características habituales, pero comienzan a presentar un número frecuente de brotes, intervalos cortos entre ellos, mayor discapacidad, o mayor número de lesiones; lo cual guarda relación con una mala respuesta al tratamiento. Es el escenario más común de alta actividad, donde el tratamiento inicia con terapias de moderada eficacia, y se valora la necesidad del cambio a drogas de alta eficacia empleando, por ejemplo, el Score de Río que valora la probabilidad de una mala respuesta según lo que acontece en el primer año de tratamiento con Interferones ${ }^{[9]}$
Escenario 2: Desde el inicio de la enfermedad. Sucede cuando la alta actividad de la enfermedad se evidencia en etapas precoces, con un primer brote con una elevada carga de lesiones en la primera RM, brote con compromiso multifocal (motores, infratentoriales o medulares), pronta evidencia de agujeros negros, lesiones de mayor tamaño, más de una lesión captadora de contraste, y se aprecian signos precoces de atrofia cortical (Figura 2); entre otros factores de riesgo descritos en la Tabla 2. Esta forma requiere un rápido control de la enfermedad, por lo que la respuesta al tratamiento con drogas de moderada eficacia será mala desde el inicio y se debe pasar al uso de drogas de alta eficacia.

\section{TRATAMIENTO DEL PACIENTE CON ALTA ACTIVIDAD}

Las guías de tratamiento en EM dan recomendaciones generales para 1) el inicio del tratamiento, 2) el cambio de tratamiento a otra droga de primera línea (switch), 3) el cambio a drogas de alta eficacia (escalamiento), y 4) cuándo suspender el tratamiento $[5,6,20,29,30]$. Sobre el tratamiento de la EM de alta actividad son claros sobre el uso de drogas de alta eficacia en el escenario 1 (durante el seguimiento de la enfermedad). Sin embargo, en el escenario 2 (desde el inicio de la enfermedad), a pesar de la evidencia disponible, son más conservadores, recomendando ser juiciosos para el uso de drogas de alta eficacia, en particular por el cuidado ante los efectos adversos ${ }^{[6,14]}$.

Son los ensayos clínicos que valoraron el uso de drogas de alta eficacia los que incluyen como una indicación las formas de EMRR de alta actividad que no han recibido tratamiento previo ${ }^{[27,28]}$. Ello se sustenta en la mala respuesta que tienen a las drogas de primera línea y el tiempo que se pierde en el control adecuado de la enfermedad. Como resultado, el inicio precoz de drogas de alta eficacia ha demostrado mejores resultados en términos clínicos y de RM al compararlos con los Interferones ${ }^{[31]}$, así como en el control de la enfermedad (NEDA-3 o NEDA-4) ${ }^{[1,17]}$. Por eso los algoritmos de tratamiento en EM dan la opción del inicio precoz de drogas de alta eficacia en pacientes con alta actividad ${ }^{[20,29]}$, tal como lo proponemos en la Figura 3.

Dentro de las opciones terapéuticas de alta eficacia (Tabla 2), se dispone del Fingolimod como droga vía oral diaria, y del Natalizumab como medicación endovenosa que se administra mensualmente. A pesar de la mayor eficacia que tiene el Fingolimod en comparación a los interferones (NEDA-3 a los 2 años de 42 y $35 \%$ respectivamente, $p<0,016)^{[32]}$, algunos autores la consideran como una droga de moderada eficacia. Un estudio observacional que comparó la eficacia entre Fingolimod y Natalizumab no encontró diferencias en cuanto a la actividad clínica, sin embargo, la evaluación del perfil en imágenes de RM mostró una mayor eficacia para Natalizumab ${ }^{[33]}$. Otro estudio observacional en pacientes nuevos con alta actividad (Escenario 2) mostró que el NEDA-3 a los 2 años en pacientes con Natalizumab fue de $75 \%$, con Fingolimod de $67 \%$ y con Interferones (administrados a altas frecuencias y altas dosis) de $40 \%{ }^{[32]}$. 


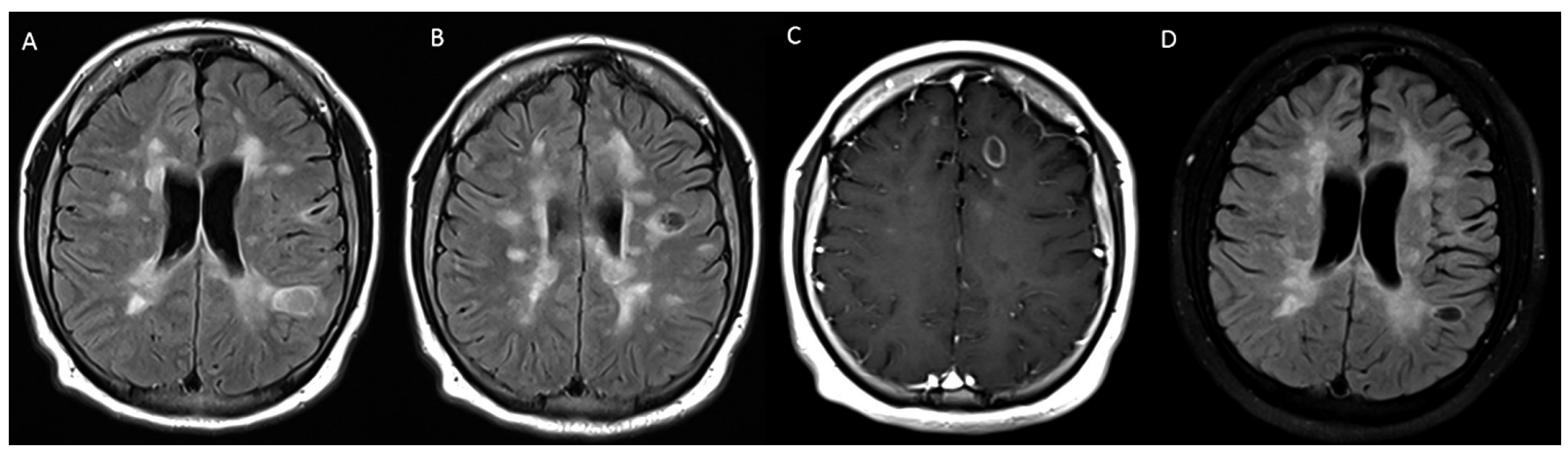

Figura 2. Paciente con esclerosis múltiple de alta actividad desde un inicio.

Resonancia magnética (RM) de varón de 14 años con tres meses de enfermedad, con dos brotes clínicos en ese periodo. En el protocolo T2-FLAIR (A y B) se aprecian múltiples imágenes ovoideas, incluso confluentes, perpendiculares a cuerpo calloso, al lado de otras cortico-yuxtacorticales, en T1 con Gadolineo (C) se aprecia captación de contraste en anillo completo y otras áreas de captación de contraste. A los 6 meses había presentado otro brote más, en la RM cerebral se aprecia signos indirectos de atrofia (mayor pronunciación de surcos y cisuras, ampliación del espacio de los ventrículos laterales). En otros planos también tenía alta carga de lesiones a nivel infratentorial y medular, todos sus brotes fueron multifocales con más de una lesión que realza contraste.

Natalizumab ha mostrado un mejor control de la actividad y disminución de la discapacidad a los 3 años en pacientes que no han recibido tratamiento y tienen menos de 1 año de enfermedad. A pesar de las ventajas clínicas, el riesgo de leucoencefalopatía multifocal progresiva (LEMP) por virus JC ha llevado a tener cuidado en su uso ${ }^{[34]}$. Son tres los factores que incrementan el riesgo de LEMP: el tiempo de tratamiento con Natalizumab, tratamiento inmunosupresor previo, y los niveles de títulos para el virus JC. En los pacientes con virus JC negativo al inicio, el riesgo de LEMP es <1:10 000; aquellos sin tratamiento inmunosupresor previo, con más de 24 meses de tratamiento y un título de virus $\mathrm{JC}<0,9$ el riesgo es de 1:748; mientas que pacientes con inmunosupresión previa y más de 24 meses de tratamiento el riesgo es 1:31 [35]. En ese sentido, es posible mantener el tratamiento por más de 24 meses, pero se debe indicar a los pacientes el riesgo de LEMP según cada caso, realizar un seguimiento más cercano con RM (cada 3 meses), e indicar las opciones de cambio, que pueden ser Fingolimod o Alemtuzumab como drogas de alta eficacia.

Alemtuzumab es una droga que presenta una mayor eficacia, aunque su perfil de seguridad, relativa poca experiencia de uso y elevado costo hace que se recomiende en casos más activos ${ }^{[6]}$. Sin embargo, en los estudios realizados incluyeron pacientes nuevos con una clara evidencia a favor de Alemtuzumab. Por ejemplo, el estudio CAMMSS223 mostró una mejora en la discapacidad a nivel sensorial, piramidal y cerebeloso a los 3 años al compararla con interferón ${ }^{[19]}$. También, en el estudio CARE-MS II se encontró que en pacientes nuevos la tasa anual de brotes fue de 0,16 en los 4 años de seguimiento, resultados significativamente mejores que con Interferón ${ }^{[36]}$. Por todo ello, es probable que dentro de poco, Alemtuzumab sea recomendada como droga de elección para formas activas.

\section{NECESIDADES EN EL PERÚ}

El tiempo es cerebro. Este axioma se extiende para todas las entidades neurológicas, sean cerebrovasculares, infecciosas o autoinmunes. A mayor retraso en el manejo, mayor pérdida neuronal, es decir, menor capacidad de la persona para relacionarse, comprender, aprender, actuar, o ejercer independientemente sus actividades de vida diaria.

En EM, el tiempo que se dispone para garantizar que un paciente tendrá menos discapacidad, es decir, para desviar el curso natural de su enfermedad, es de dos años ${ }^{[14,15]}$. Por ello, debe ser el objetivo de todo clínico identificar precozmente a pacientes con riesgo de EM o en estadios iniciales para facilitar su diagnóstico e inicio precoz del tratamiento, individualizando el caso según la magnitud de la enfermedad. Sin embargo, existen varios tipos de demoras: al buscar atención médica, en el diagnóstico, en el inicio del tratamiento, y para el cambio de tratamiento.

En el Perú, al igual que en otros países vecinos, al tener una menor prevalencia y dificultades para el acceso a estudios de neuroimágenes con RM (sean geográficas o económicas), hace aún más difícil la correcta valoración de la EM, dado que son pocos los especialistas que ven con frecuencia estos pacientes, donde las decisiones terapéuticas deben ser individualizadas y muchas veces son difíciles por la complejidad clínica e imagenológica que debe ser evaluada en una línea de tiempo a veces muy extensa. Así, es común un retraso de varios meses desde el primer brote clínico hasta el diagnóstico, momento en el que el paciente tiene una mayor carga de lesiones o ya ha tenido varios brotes.

Luego que un paciente llega a ser diagnosticado de EM, la siguiente dificultad está en poder recibir la medicación adecuada 


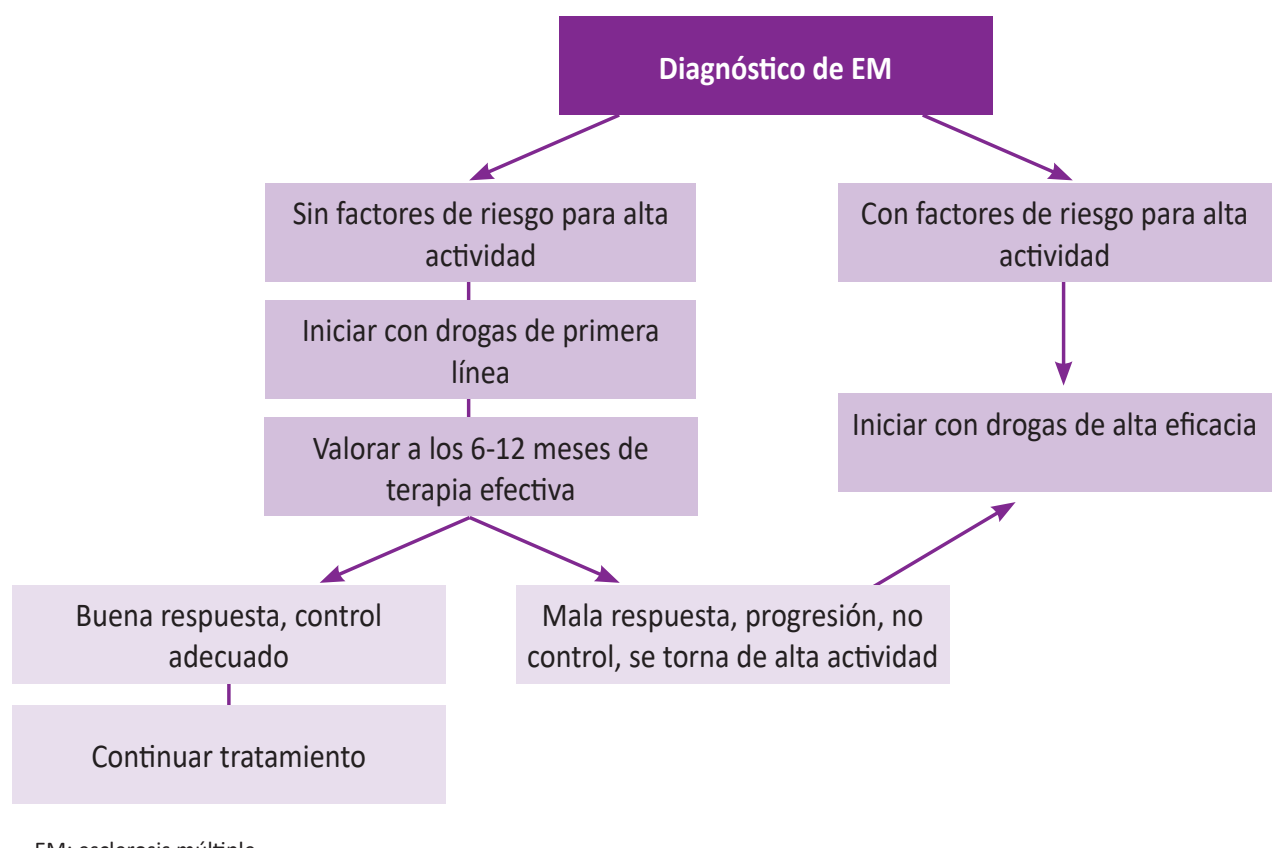

Figura 3. Algoritmo de tratamiento en esclerosis múltiple de alta actividad.

y oportuna. El proceso yace en la vía administrativa, y dado que varios de los medicamentos están fuera del petitorio farmacológico o son de costo elevado, deben ser presentados a un comité farmacológico que puede no valorar adecuadamente el historial clínico que llevó a esta decisión, además que este proceso es lento inclusive para las drogas de primera línea. Como resultado tenemos que en los establecimientos públicos del Perú ocurre lo siguiente:

1) solo se dispone del uso de Interferón o acetato de glatiramer como drogas iniciales, independiente de la actividad de la enfermedad;

2) el proceso de aprobación del uso de drogas de primera línea demora en promedio 3 meses;

3) solo se puede pasar a drogas de alta eficacia ante falla al tratamiento de las de primera línea, exigiendo su uso continuo por al menos 12 meses. No está disponible como medicamento de inicio precoz en formas de EM de alta actividad o agresivas;

4) el proceso de compra y entrega de drogas de alta eficacia demora de 4 a 6 meses, pudiendo ser observado desde el punto de vista administrativo, con pocos casos donde se ha logrado brindar el tratamiento.

Los tratamientos de alto costo en el Perú ciertamente requieren de la evaluación juiciosa de los comités a cargo para valorar su adecuada prescripción, pero estos trámites conducen a que los medicamentos de alta eficacia lleguen a los pacientes luego del segundo año de enfermedad, momento en el cual su efectividad está disminuida notablemente y el paciente ya presenta una mayor discapacidad.
Por ello, se recomienda que en pacientes con EM de alta actividad, se disponga de medicamentos de alta eficacia dentro del petitorio farmacológico de los establecimientos públicos en el Perú, para brindar eficazmente un tratamiento precoz. Una propuesta para facilitar el inicio precoz con terapia de alta eficacia es el uso del algoritmo de EM con alta actividad que proponemos en la Figura 3.

\section{CONCLUSIONES}

No existe un consenso para EM de alta actividad, pero esta se sustenta en el número de brotes, sus características, el número de lesiones, la presencia de agujeros negros, atrofia cerebral, entre otras. Pudiendo distinguir la alta actividad durante el curso de la enfermedad o desde etapas precoces. Se sabe que de no controlar la enfermedad en los primeros dos años, no se logrará controlar eficazmente su curso y la posibilidad de tener una mayor discapacidad a corto plazo es mayor. Por tanto, es indispensable la identificación precoz de casos de EM de alta actividad e iniciar rápidamente un tratamiento adecuado. En el Perú, además de las dificultades clínicas, también se tienen dificultades administrativas que deben ser superadas. Por ello se recomienda la necesidad de un mayor arsenal terapéutico y la posibilidad de iniciar de forma precoz con drogas de alta eficacia.

Fuente de financiamiento: Los autores declaran no haber recibido ninguna financiación para la realización de este trabajo.

Declaración de conflicto de intereses: Los autores declaran no tener conflicto de intereses con la publicación de este artículo. 


\section{REFERENCIAS BIBLIOGRÁFICAS}

1. Evans C, Beland SG, Kulaga S, Wolfson C, Kingwell E, Marriott J, et al. Incidence and prevalence of multiple sclerosis in the Americas: a systematic review. Neuroepidemiology. 2013;40(3):195-210.

2. Koch-Henriksen N, Sorensen PS. The changing demographic pattern of multiple sclerosis epidemiology. Lancet Neurol. 2010;9(5):520-32.

3. Portillo-Vallenas R, Rojas-Huerto E, Inca-Parra J. Control clínico de pacientes con esclerosis múltiple tratados con interferón beta en un hospital peruano. Rev Neurol. 2005;41(9):575-6.

4. Ziemssen T, De Stefano N, Pia Sormani M, Van Wijmeersch B, Wiendl $\mathrm{H}$, Kieseier BC. Optimizing therapy early in multiple sclerosis: An evidence-based view. Mult Scler Relat Disord. 2015;4(5):460-9.

5. Freedman MS, Selchen D, Arnold DL, Prat A, Banwell B, Yeung M, et al. Treatment optimization in MS: Canadian MS Working Group updated recommendations. Can J Neurol Sci. 2013;40(3):307-23.

6. Scolding N, Barnes D, Cader S, Chataway J, Chaudhuri A, Coles A, et al. Association of British Neurologists: revised (2015) guidelines for prescribing disease-modifying treatments in multiple sclerosis. Pract Neurol. 2015;15(4):273-9.

7. Lublin FD, Reingold SC, Cohen JA, Cutter GR, Sorensen PS, Thompson $\mathrm{AJ}$, et al. Defining the clinical course of multiple sclerosis: the 2013 revisions. Neurology. 2014;83(3):278-86.

8. Kurtzke JF. Rating neurologic impairment in multiple sclerosis: an expanded disability status scale (EDSS). Neurology. 1983;33(11):1444-52.

9. Rio J, Nos C, Tintore M, Tellez N, Galan I, Pelayo R, et al. Defining the response to interferon-beta in relapsing-remitting multiple sclerosis patients. Ann Neurol. 2006;59(2):344-52.

10. Kappos L, De Stefano N, Freedman MS, Cree BAC, Radue E-W, Sprenger $\mathrm{T}$, et al. Inclusion of brain volume loss in a revised measure of 'no evidence of disease activity' (NEDA-4) in relapsing-remitting multiple sclerosis. Mult Scler. 2016;22(10):1297-305.

11. Filippi M, Rocca MA, Ciccarelli O, De Stefano N, Evangelou N, Kappos $\mathrm{L}$, et al. MRI criteria for the diagnosis of multiple sclerosis: MAGNIMS consensus guidelines. Lancet Neurol. 2016;15(3):292-303.

12. Marziniak M, Ghorab K, Kozubski W, Pfleger C, Sousa L, Vernon K, et al. Variations in multiple sclerosis practice within Europe-Is it time for a new treatment guideline? Mult Scler Relat Disord. 2016;8:35-44.

13. Rotstein DL, Healy BC, Malik MT, Chitnis T, Weiner HL. Evaluation of no evidence of disease activity in a 7-year longitudinal multiple sclerosis cohort. JAMA Neurol. 2015;72(2):152-8.

14. Ziemssen T, Derfuss T, de Stefano N, Giovannoni G, Palavra F, Tomic $D$, et al. Optimizing treatment success in multiple sclerosis. J Neurol. 2016;263(6):1053-65.

15. Bermel RA, You X, Foulds $P$, Hyde R, Simon JH, Fisher E, et al. Predictors of long-term outcome in multiple sclerosis patients treated with interferon beta. Ann Neurol. 2013;73(1):95-103.

16. Kappos L, Polman CH, Freedman MS, Edan G, Hartung HP, Miller $\mathrm{DH}$, et al. Treatment with interferon beta- $1 \mathrm{~b}$ delays conversion to clinically definite and McDonald MS in patients with clinically isolated syndromes. Neurology. 2006;67(7):1242-9.

17. Wiendl H, Meuth SG. Pharmacological Approaches to Delaying Disability Progression in Patients with Multiple Sclerosis. Drugs. 2015;75(9):947-77.

18. Kappos L, Radue EW, O'Connor P, Polman C, Hohlfeld R, Calabresi $\mathrm{P}$, et al. A placebo-controlled trial of oral fingolimod in relapsing multiple sclerosis. N Engl J Med. 2010;362(5):387-401.

19. Fox EJ, Wynn D, Coles AJ, Palmer J, Margolin DH. Alemtuzumab improves neurological functional systems in treatment-naive relapsing-remitting multiple sclerosis patients. J Neurol Sci. 2016;363:188-94.

20. Comini-Frota ER, Vasconcelos CC, Mendes MF. Guideline for multiple sclerosis treatment in Brazil: Consensus from the Neuroimmunology Scientific Department of the Brazilian Academy of Neurology. Arq Neuropsiquiatr. 2017;75(1):57-65.

21. Montalban X, Hauser SL, Kappos L, Arnold DL, Bar-Or A, Comi G, et al. Ocrelizumab versus Placebo in Primary Progressive Multiple Sclerosis. N Engl J Med. 2017;376(3):209-20.

22. Freedman MS, Rush CA. Severe, highly active, or aggressive multiple sclerosis. Continuum (Minneap Minn). 2016;22(3):761-84.

23. Sayao A-L, Devonshire V, Tremlett H. Longitudinal follow-up of "benign" multiple sclerosis at 20 years. Neurology. 2007;68(7):496-500.

24. Capello E, Mancardi GL. Marburg type and Balò's concentric sclerosis: rare and acute variants of multiple sclerosis. Neurol Sci. 2004;25(4):s361-s3.

25. Menon S, Shirani A, Zhao Y, Oger J, Traboulsee A, Freedman MS, et al. Characterising aggressive multiple sclerosis. J Neurol Neurosurg Psychiatry. 2013;84(11):1192-8.

26. Rush CA, MacLean HJ, Freedman MS. Aggressive multiple sclerosis: proposed definition and treatment algorithm. Nat Rev Neurol. 2015;11(7):379-89.

27. Hutchinson M, Kappos L, Calabresi PA, Confavreux C, Giovannoni G, Galetta SL, et al. The efficacy of natalizumab in patients with relapsing multiple sclerosis: subgroup analyses of AFFIRM and SENTINEL. J Neurol. 2009;256(3):405-15.

28. Devonshire V, Havrdova E, Radue EW, O'Connor P, Zhang-Auberson $\mathrm{L}$, Agoropoulou C, et al. Relapse and disability outcomes in patients with multiple sclerosis treated with fingolimod: subgroup analyses of the double-blind, randomised, placebo-controlled FREEDOMS study. Lancet Neurol. 2012;11(5):420-8.

29. Kamm CP, Uitdehaag BM, Polman CH. Multiple sclerosis: current knowledge and future outlook. Eur Neurol. 2014;72(3-4):132-41.

30. Marziniak M, Ghorab K, Kozubski W, Pfleger C, Sousa L, Vernon K, et al. Variations in multiple sclerosis practice within Europe - Is it time for a new treatment guideline? Mult Scler Relat Disord. 2016;8:35-44

31. Khatri B, Barkhof F, Comi G, Hartung HP, Kappos L, Montalban $X$, et al. Comparison of fingolimod with interferon beta-1a in relapsing-remitting multiple sclerosis: a randomised extension of the TRANSFORMS study. Lancet Neurol. 2011;10(6):520-9.

32. Prosperini L, Saccà F, Cordioli C, Cortese A, Buttari F, Pontecorvo $S$, et al. Real-world effectiveness of natalizumab and fingolimod compared with self-injectable drugs in non-responders and in treatment-naïve patients with multiple sclerosis. J Neurol. 2017;264(2):284-94.

33. Koch-Henriksen N, Magyari M, Sellebjerg F, Soelberg Sørensen P. A comparison of multiple sclerosis clinical disease activity between patients treated with natalizumab and fingolimod. Mult Scler. 2016;23(2):234-41.

34. Nicholas JA, Racke MK, Imitola J, Boster AL. First-line natalizumab in multiple sclerosis: rationale, patient selection, benefits and risks. Ther Adv Chronic Dis. 2013;5(2):62-8.

35. Schwab N, Schneider-Hohendorf T, Melzer N, Cutter G, Wiendl H. Natalizumab-associated PML: Challenges with incidence, resulting risk, and risk stratification. Neurology. 2017;88(12):1197-205.

36. Havrdova E, Horakova D, Kovarova I. Alemtuzumab in the treatment of multiple sclerosis: key clinical trial results and considerations for use. Ther Adv Neurol Disord. 2015;8(1):31-45. 